lustitia Socialis. Revista Arbitrada de Ciencias Jurídicas.

Año V. Vol. V. N³. Edición Especial. 2020-III:

Universidad Regional Autónoma de los Andes

Hecho el depósito de Ley: FA2016000064

ISSN: 2542-3371

FUNDACIÓN KOINONIA (F.K). Santa Ana de Coro, Venezuela

Iyo Alexis Cruz-Piza; Lady Carolina Flores-Córdova

http://dx.doi.org/10.35381/racji.v5i3.1076

\title{
Seguridad jurídica y medios de prueba para impugnar las contravenciones de tránsito
}

Legal certainty and means of proof to challenge traffic contractions

\author{
lyo Alexis Cruz-Piza \\ ub.iyocruz@uniandes.edu.ec \\ Universidad Regional Autónoma de los Andes, Babahoyo \\ Ecuador \\ https://orcid.org/0000-0002-2655-172X \\ Lady Carolina Flores-Córdova \\ db.ladycfc49@uniandes.edu.ec- \\ Universidad Regional Autónoma de los Andes, Babahoyo \\ Ecuador
}

Recibido: 31 de octubre de 2020

Revisado: 10 de octubre de 2020

Aprobado: 05 de diciembre de 2020

Publicado: 10 de diciembre de 2020 
lustitia Socialis. Revista Arbitrada de Ciencias Jurídicas.

Año V. Vol. V. N³. Edición Especial. 2020-III:

Universidad Regional Autónoma de los Andes

Hecho el depósito de Ley: FA2016000064

ISSN: 2542-3371

FUNDACIÓN KOINONIA (F.K). Santa Ana de Coro, Venezuela

Iyo Alexis Cruz-Piza; Lady Carolina Flores-Córdova

\title{
RESUMEN
}

En el siguiente trabajo de revisión se pretende analizar el proceso № 12282-2019-01005, a fin de proponer una normativa que especifique la jurisdicción y competencia de los agentes de tránsito municipal y de la Comisión de tránsito del Ecuador. Desde la perspectiva cuantitativa, de tipo descriptiva se complementa por un diseño documentalbibliográfico, abordando el método analítico enfocándose en el estudio de aspectos particulares, examinados en el análisis de contenido escrito, mediante lo analíticosintético. Se utilizó una muestra por conveniencia, de una población de 1500 abogados inscritos en el foro de abogados de Babahoyo, se obtuvo una muestra de 206 abogados, a los cuales se les aplicó un cuestionario, conformado por 5 interrogantes. Se pudo concluir que en el proceso existió la falta de valoración de las pruebas por parte del juez de garantías penales en la audiencia de juzgamiento por contravenciones de tránsito. Recomendando que se debe realizar una revisión y mejorar en los procedimientos futuros.

Descriptores: Administración de justicia; mediación; sistemas jurídicos. (Palabras tomadas del tesauro UNESCO).

\begin{abstract}
In the following review work, it is intended to analyze the process $N^{\circ} 12282-2019-01005$, in order to propose a regulation that specifies the jurisdiction and competence of the municipal traffic agents and the Ecuadorian Traffic Commission. From a quantitative perspective, of a descriptive type, it is complemented by a documentary-bibliographic design, addressing the analytical method focusing on the study of particular aspects, examined in the analysis of written content, through the analytical-synthetic. A convenience sample was used, out of a population of 1500 lawyers registered in the Babahoyo Lawyers Forum, a sample of 206 lawyers was obtained, to whom a questionnaire was applied, consisting of 5 questions. It was concluded that in the process there was a lack of assessment of the evidence by the criminal guarantees judge in the trial hearing for traffic violations. Recommending that a review and improvement should be performed in future procedures.
\end{abstract}

Descriptors: Justice administration; mediation; legal systems. (Words taken from the UNESCO thesaurus). 


\author{
lustitia Socialis. Revista Arbitrada de Ciencias Jurídicas. \\ Año V. Vol. V. №3. Edición Especial. 2020-III: \\ Universidad Regional Autónoma de los Andes \\ Hecho el depósito de Ley: FA2016000064 \\ ISSN: 2542-3371 \\ FUNDACIÓN KOINONIA (F.K). Santa Ana de Coro, Venezuela \\ Iyo Alexis Cruz-Piza; Lady Carolina Flores-Córdova
}

\title{
INTRODUCCIÓN
}

En toda acción jurídica debe existir una justa acción por parte de los órganos garantes de cumplir y hacer cumplir la constitución y sus respectivas leyes y normas, sin perjuicio de los conciudadanos, garantizando el debido proceso el cual debe buscar en la mayor medida viable fallos legales ecuánimes e imparciales en las disputas legales, respetando y velando por los derechos de los sujetos procesales,

en concordancia con el artículo 426 de la Constitución de la República del Ecuador, cuya disposición se menciona a tenor:

Articula 426, Todas las personas, autoridades e instituciones están sujetas a la Constitución. Las Juezas y Jueces, autoridades administrativas y servidoras y servidores públicos, aplicaran directamente las normas constitucionales y las previstas en los instrumentos internacionales de derechos humanos siempre que sean más favorables a las establecidas en la Constitución, aunque las partes no las invoquen expresamente. Los derechos consagrados en la Constitución y los instrumentos internacionales de derechos Humanos serán de inmediato cumplimiento y de aplicación. No podrá alegarse falta de ley y desconocimiento de las normas para justificar la vulneración de los derechos y garantías establecidos en la Constitución, para desechar la acción interpuesta en su defensa ni para negar el reconocimiento de tales derechos.

En consideración al artículo anterior, se enuncia que las garantías en todo proceso judicial tienen sus bases en la protección nacional e internacional y el principio de supremacía. Sin embargo, ocurren hechos en los cuales son vulneradas ciertas reglas y procedimientos claramente establecidos, las impugnaciones de tránsito son el pan de cada día, de las personas que concurren en alguna infracción contravencional y muchas veces se consideran insatisfechas sus pretensiones, tomando en consideración lo que examinaremos en el caso propuesto, no siempre se lleva un criterio uniforme al momento de dictar una resolución. Sin embargo, el Estado ecuatoriano debe garantizar el debido 


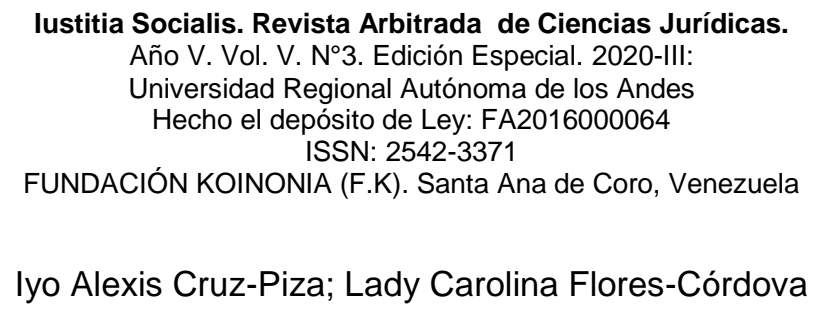

proceso tutelar efectivo, de acuerdo a lo manifestado por Cevallo-Sánchez \& AlvaradoMoncada (2018):

Uno de los pilares fundamentales para el ejercicio de los derechos y que consta en el texto constitucional, es la responsabilidad del Estado ecuatoriano, por transgredir el derecho a la tutela judicial efectiva y las reglas del debido proceso, teniendo en consideración que es un derecho humano que tienen todas las personas de gozar las garantías fundamentales de una justicia trasparente y equitativa, y por lo tanto es su deber velar porque los ciudadanos gocen de esta garantía fundamental.(p.169)

En ente discurrir jurídico revisaremos lo que plantea el Código Orgánico Integral Penal, en la materia, al respecto el Articulo 641 especifica lo siguiente:

Procedimiento expedito. Las contravenciones penales y de tránsito serán susceptibles de procedimiento expedito. El procedimiento se desarrollará en una sola audiencia ante la o el juzgador competente la cual se regirá por las reglas generales previstas en este Código. En la audiencia, la víctima y el denunciado si corresponde podrán llegar a una conciliación, salvo el caso de violencia contra la mujer o miembros del núcleo familiar. El acuerdo se pondrá en conocimiento de la o el juzgador para que ponga fin al proceso.

Así mismo el artículo 644 establece el procedimiento de impugnación de las citaciones de tránsito que deben seguir las personas que se sientan perjudicadas. Estos procedimientos deben adecuarse también a los principios y derechos reconocidos, tanto en la Constitución de la República del Ecuador como en el mismo Código Orgánico Integral Penal.

En el presente trabajo investigativo se realiza el análisis del Proceso No. 12282-201901005, en donde el juzgador emitió su sentencia en razón de la incompetencia que supuestamente tiene la Comisión de Tránsito del Ecuador, dentro del territorio en donde la boleta de citación fue emitida. Se examinará de acuerdo a la competencia que tiene la Comisión de Transito del Ecuador, análisis de la prueba presentada, la motivación del juez y su decisión. 


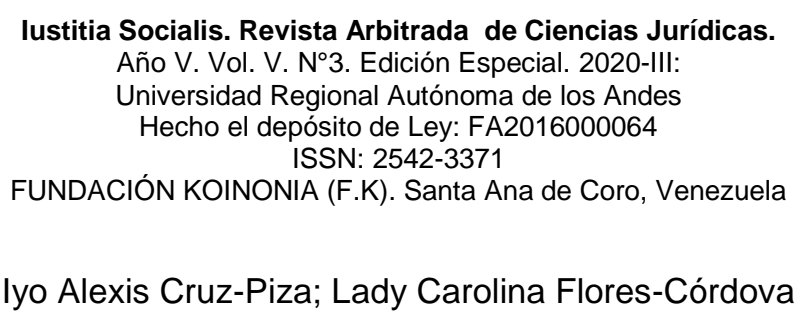

En este recurso de revisión veamos que nos indica el Articulo 55 del Código Orgánico de Organización Territorial, Autonomía y Descentralización (2010), establece lo siguiente: "Los gobiernos autónomos descentralizados municipales tendrán las siguientes competencias exclusivas sin perjuicio de otras que determine la ley; f) Planificar, regular y controlar el tránsito y el transporte terrestre dentro de su circunscripción cantonal".

Por todo lo expuesto es de suma importancia establecer cuáles son las competencias territoriales de los Agentes de Tránsito Municipal y la Comisión de Tránsito del Ecuador, para identificar la congruencia de la sentencia emitida por el juez en el proceso antes mencionado.

Los Hechos. En la Ciudad de Babahoyo, son comunes las infracciones de tránsito, las sanciones y las inconformidades, tanto así que el diario La Hora expresa: Decenas de conductores terminan siendo sancionados por la Agencia de Tránsito Municipal (ATM) Babahoyo, una vez que entraron en marcha los fotocensores y las fotomultas.

Según datos de la ATM, desde el primero de septiembre que entraron en vigencia la operatividad y funcionamiento de estos dispositivos, hasta el pasado martes 10 de septiembre, un total de 1.830 vehículos y conductores fueron detectados infringiendo la ley. Lo que en promedio de 180 personas a diario son multadas en la capital fluminense. De hecho, estos equipos de control registran un mayor número de emisión de multas que los mismos agentes civiles de tránsito, puesto que dicho personal, desde el primero de agosto (que empezaron a citar) hasta el mismo 10 de septiembre, han multado a 1.227 conductores por distintas infracciones.

Algunos conductores han mostrado su malestar por la entrada en vigencia de los fotocensores, pues refieren que los valores son excesivos, al respecto manifiestan:

"A veces tenemos muchas situaciones por cumplir y andamos contra el tiempo. Es allí que sin querer terminamos cometiendo la infracción y se nos castiga con multas de hasta 400 dólares", dijo el taxista Joselo Ramírez.

De igual forma, Joffre López, quien conducía un automóvil Aveo Family, fue amonestado con 394 dólares tras exceder el límite de velocidad fuera del rango moderado en la vía 
lustitia Socialis. Revista Arbitrada de Ciencias Jurídicas.

Año V. Vol. V. N³. Edición Especial. 2020-III:

Universidad Regional Autónoma de los Andes

Hecho el depósito de Ley: FA2016000064

ISSN: 2542-3371

FUNDACIÓN KOINONIA (F.K). Santa Ana de Coro, Venezuela

Iyo Alexis Cruz-Piza; Lady Carolina Flores-Córdova

Jujan - Babahoyo, frente a El Paseo Shopping Center. Ahora no le queda más que pagar dicho valor para poder matricular el vehículo y tenerlo en regla.

Para la verificación de alguna multa las citaciones pueden revisarse en el enlace www.babahoyo.gob.ec y acceder a la opción ATM, donde ingresando sus datos podrán revisar si tienen o no infracciones, también acudiendo a la sede de la agencia. En relación a las trasgresiones el jefe operativo de la ATM - Babahoyo, Saúl Pezo, indicó que todas las personas que resultan multadas, tendrán tres días para apelar, pero si no lo hace dentro de ese tiempo, se entenderá que el conductor o propietario del vehículo ha aceptado la multa. Manifiesta, además, que "la sanción no es una camisa de fuerza, es una notificación, la cual tiene un amparo de la propia Constitución para el ciudadano, que si no está de acuerdo o cree que le han vulnerado sus derechos, tiene la oportunidad de solicitar a un juez la apelación correspondiente. En una audiencia se determinará si tiene o no la razón", explicó Pezo. (Hora, 2019)

Por todo lo expuesto se pretende analizar el proceso № 12282-2019-01005 a fin de proponer una normativa que especifique la jurisdicción y competencia de los agentes de tránsito municipal y de la Comisión de tránsito del Ecuador.

\section{MÉTODO}

El estudio desde la perspectiva cuantitativa, de tipo descriptiva se complementa por un diseño documental-bibliográfico, (Parela-Stracuzzi \& Martins-Pestana, 2012) que permitió la recogida de información extraída de los diferentes textos jurídicos abordando el método analítico enfocándose en el estudio de aspectos particulares, examinados en el análisis de contenido escrito, mediante lo analítico-sintético (Bernal-Torres, 2006), obteniendo nuevos conocimientos de la descomposición mental en varios elementos. Constantemente es significativo ver el pasado para edificar el presente y no perder de vista el fututo. La ciencia es un cuerpo sistemático y estructurado, resulta provechoso localizar, conseguir y examinar estudios, antecedentes, libros entre otros (Gómez,2006). Así mismo se, analizaron leyes, normas y teorías. Se utilizó un muestro por conveniencia, 


\section{lustitia Socialis. Revista Arbitrada de Ciencias Jurídicas. \\ Año V. Vol. V. №3. Edición Especial. 2020-III: \\ Universidad Regional Autónoma de los Andes \\ Hecho el depósito de Ley: FA2016000064 ISSN: 2542-3371 \\ FUNDACIÓN KOINONIA (F.K). Santa Ana de Coro, Venezuela \\ Iyo Alexis Cruz-Piza; Lady Carolina Flores-Córdova}

de una población de 1500 abogados inscritos en el foro de abogados de la ciudad de Babahoyo, Capital de la Provincia de Los Ríos, de la cual se obtuvo una muestra de 206 abogados, a los cuales se les aplicó un cuestionario y se obtuvieron datos que nos dio como resultado el porcentaje de las personas que conocen y cuantas desconocen de este principio y la injerencia en el cuerpo legal.

\section{DISCUCIÓN}

A continuación, se presenta los resultados obtenidos de la encuesta, realizada a profesionales del derecho inscritos en el foro de abogados de Los Ríos.

\section{Tabla $\mathbf{N}^{\circ} 1$.}

¿Ha intervenido en impugnaciones de tránsito?

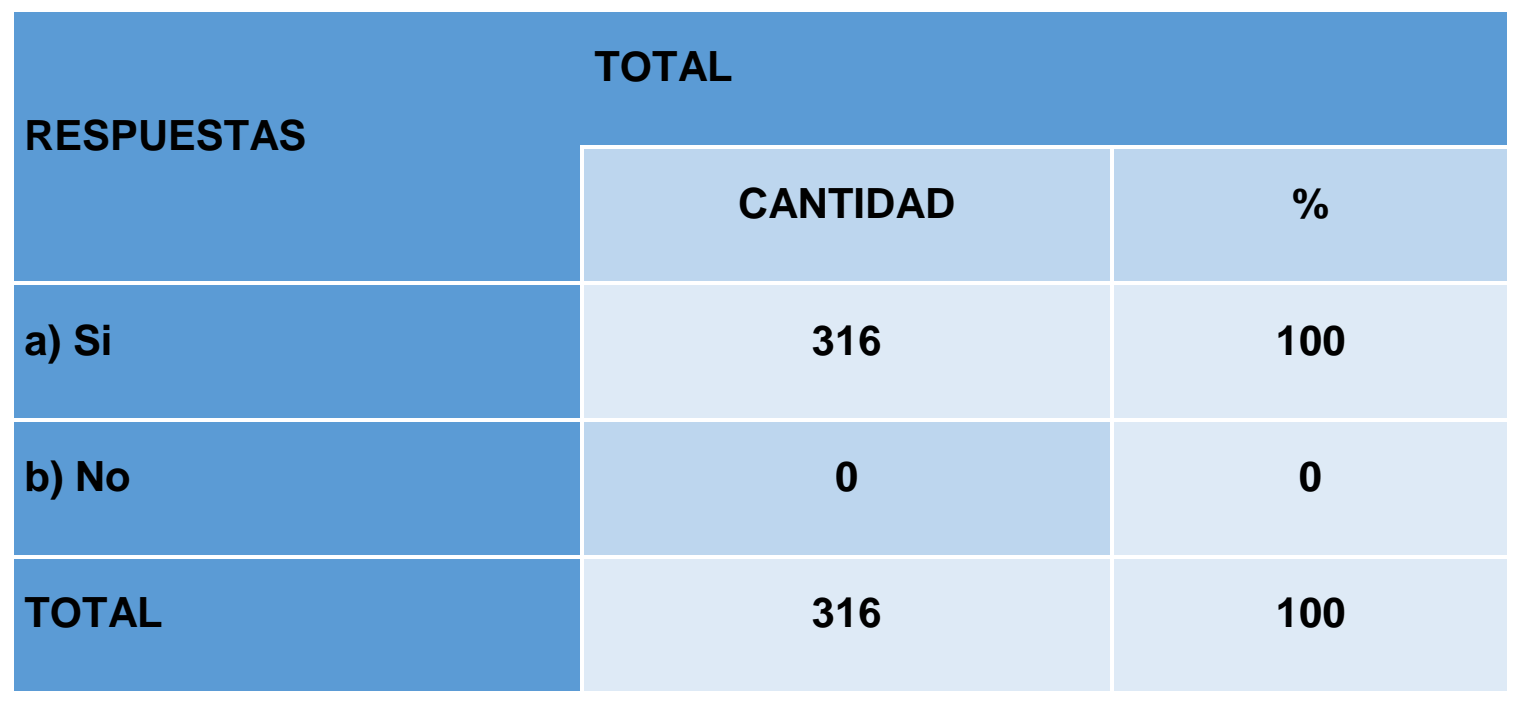

Fuente: Cruz \& Flores (2020)

Se observa en la tabla $\mathrm{N}^{\circ} 1$ los resultados obtenidos, en donde el total de profesionales del derecho responden en un $100 \%$ que si habían participado al menos en una impugnación de tránsito. 
lustitia Socialis. Revista Arbitrada de Ciencias Jurídicas.

Año V. Vol. V. №3. Edición Especial. 2020-III:

Universidad Regional Autónoma de los Andes

Hecho el depósito de Ley: FA2016000064

ISSN: 2542-3371

FUNDACIÓN KOINONIA (F.K). Santa Ana de Coro, Venezuela

Iyo Alexis Cruz-Piza; Lady Carolina Flores-Córdova

Tabla 2.

¿Existe coherencia, seguridad jurídica y criterios jurídicos al momento de aplicar una sanción por parte del juzgador de una contravención?

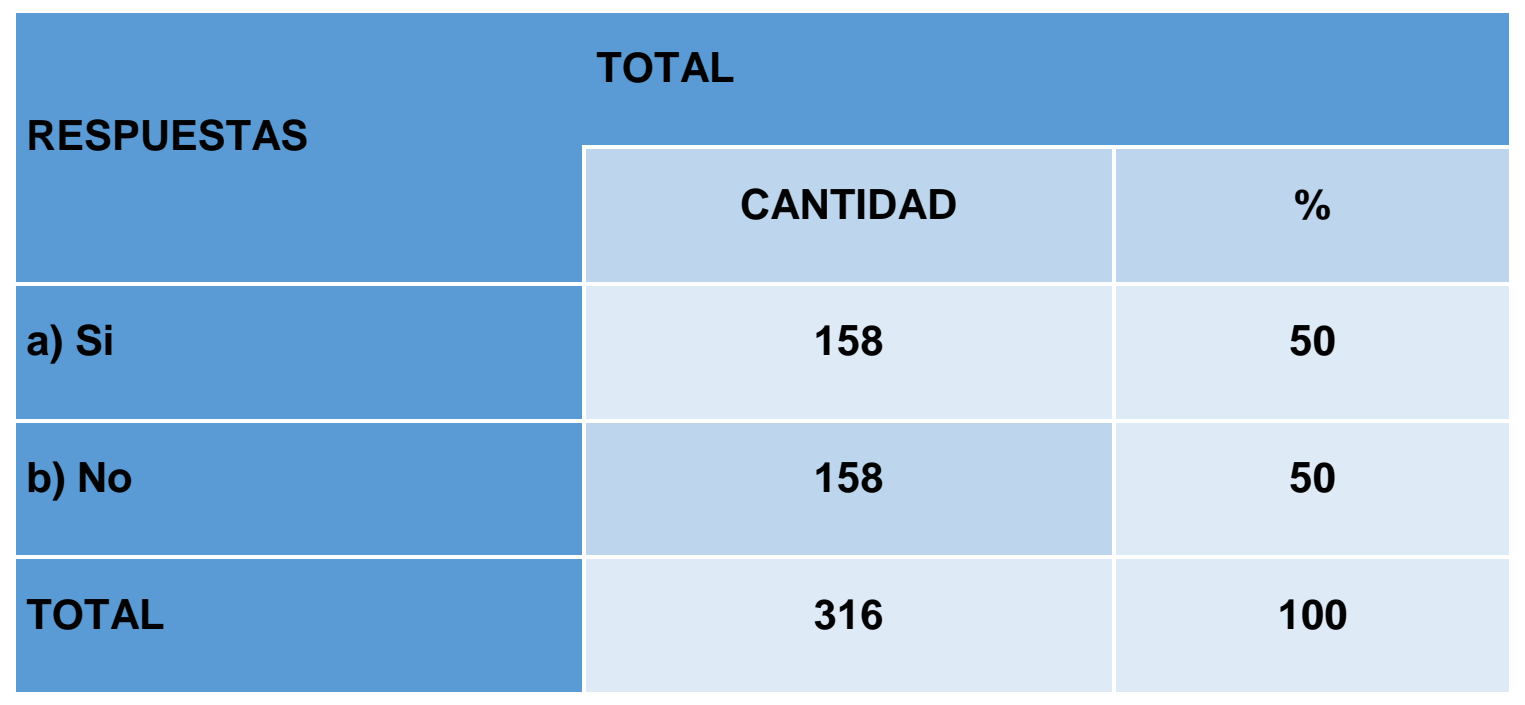

Fuente: Cruz \& Flores (2020).

Se determina un criterio dividido, ya que algunos abogados piensan que no existe criterio jurídico suficiente al momento de plantear una sanción por lo que resta credibilidad a los operadores de justicia. 
lustitia Socialis. Revista Arbitrada de Ciencias Jurídicas.

Año V. Vol. V. №3. Edición Especial. 2020-III:

Universidad Regional Autónoma de los Andes

Hecho el depósito de Ley: FA2016000064

ISSN: 2542-3371

FUNDACIÓN KOINONIA (F.K). Santa Ana de Coro, Venezuela

Iyo Alexis Cruz-Piza; Lady Carolina Flores-Córdova

\section{Tabla 3.}

¿Considera usted en el proceso 12282-2019-01005 el juez de garantías penales debió ratificar la sanción?

\begin{tabular}{l|l|l|}
\multicolumn{2}{l}{ TOTAL } \\
RESPUESTAS & CANTIDAD & $\%$ \\
\cline { 2 - 3 } & & \\
\hline a) Si & 221 & 70 \\
\hline b) No & 95 & 30 \\
\hline TOTAL & 316 & 100 \\
\hline
\end{tabular}

Fuente: Cruz \& Flores (2020).

Se aprecia que el $30 \%$ de los profesionales del derecho expresaron que el juez no debió ratificar la sanción, mientras que, el otro $70 \%$ de indicaron que se debió ratificar la sanción por cuanto existía prueba suficiente del cometimiento de la infracción. 
lustitia Socialis. Revista Arbitrada de Ciencias Jurídicas.

Año V. Vol. V. №3. Edición Especial. 2020-III:

Universidad Regional Autónoma de los Andes

Hecho el depósito de Ley: FA2016000064

ISSN: 2542-3371

FUNDACIÓN KOINONIA (F.K). Santa Ana de Coro, Venezuela

Iyo Alexis Cruz-Piza; Lady Carolina Flores-Córdova

Tabla 4.

¿Considera que el juez valoró la prueba de forma correcta?

\begin{tabular}{|l|c|c|}
\multicolumn{2}{|c|}{ TOTAL } \\
\cline { 2 - 3 } RESPUESTAS & CANTIDAD & $\%$ \\
\hline a) $\mathrm{Si}$ & 0 & 0 \\
\hline b) No & 316 & 100 \\
\hline TOTAL & 316 & 100 \\
\hline
\end{tabular}

Fuente: Cruz \& Flores (2020).

De las 316 personas encuestadas, todos profesionales del derecho coinciden en que el juez no valoró de forma correcta la prueba presentada por el agente de tránsito. 


\section{lustitia Socialis. Revista Arbitrada de Ciencias Jurídicas. \\ Año V. Vol. V. №3. Edición Especial. 2020-III: \\ Universidad Regional Autónoma de los Andes \\ Hecho el depósito de Ley: FA2016000064 \\ ISSN: 2542-3371 \\ FUNDACIÓN KOINONIA (F.K). Santa Ana de Coro, Venezuela \\ Iyo Alexis Cruz-Piza; Lady Carolina Flores-Córdova}

\section{Tabla 5.}

¿Considera correcta la actuación del juez frente a la prueba presentada y a la decisión del agente de tránsito en ejercicio de sus funciones?

\begin{tabular}{|l|c|c|}
\hline \multicolumn{2}{|l|}{ TOTAL } \\
\hline RESPUESTAS & CANTIDAD & $\%$ \\
\hline a) Si & 0 & 0 \\
\hline b) No & 316 & 100 \\
\hline TOTAL & 316 & 100 \\
\hline
\end{tabular}

Fuente: Cruz \& Flores (2020).

En su totalidad los abogados, consideran incorrecta la decisión del juez y discurren que hacen inertes las acciones tomadas por los agentes de tránsito pues las sanciones a pesar de la prueba no son valoradas correctamente.

En consecuencia, una vez analizado las respuestas de los profesionales del derecho y la revisión documental se realiza el siguiente análisis los hechos fueron conocido y resuelto por la Unidad d Judicial Penal de Babahoyo, por otro lado, el juez al dictar la resolución lo hace $\sin$ los recaudos probatorios, es decir no toma en consideración la prueba. Se sostiene de un subterfugio legal para dictar la contraversión en favor de la aprensión al imputado. En este sentido, es válido lo manifestado por Marcheco-Acuña (2017): 


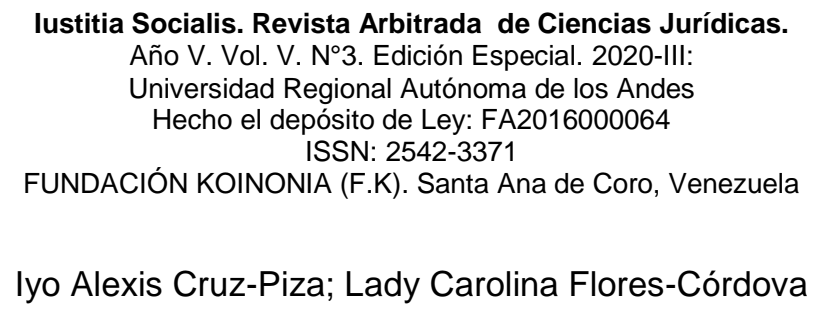

Frente a este panorama, ante la existencia de un poder público con la capacidad de actuar aun cuando haya sido puesta en cuestionamiento la legalidad de su decisión, la protección cautelar se presenta como una garantía esencial de la tutela judicial efectiva del ciudadano. (p.265)

Por todo ello, la prueba no sirve para determinar la responsabilidad que tiene la persona por cometer la infracción, existen otros medios, a los cuales el magistrado ha hecho caso omiso. Se recomienda que los jueces tengan pleno conocimiento de las competencias de los agentes de tránsito de la ATM como los de la municipalidad y esclarecer el verdadero propósito de la prueba y hacerla efectiva dentro de un proceso penal en cualquier tipo de procedimiento. Los jueces deben valorar las pruebas más aun cuando estas llevan al convencimiento de la existencia de una infracción.

Al respecto se puede mostrar lo indicado por la Corte Constitucional (2012) que el debido proceso es una demarcación para la discrecionalidad de los jueces, a quienes corresponde presidir por el mismo, definido como las garantías mínimas explícitas en la Constitución y cuyo objetivo es certificar a los sujetos judiciales resoluciones justas, sujetas a derecho. Por consiguiente, es recomendable, ampliar la visión en materia judicial, garantizando los derechos de los ciudadanos, como lo expresa Zambrano-Noles, S. (2016):

El enfoque integral hace referencia al sentido de un sistema integrado de seguridad, que abarca todos los ámbitos del ser humano y del Estado, sin dejar nada al azar. Tiene que ver con la integración de todas las esferas de la seguridad, las cuales se complementan y se integran en un solo sistema: el sistema de seguridad pública y del Estado. Ahí encontramos la seguridad internacional, la seguridad interna, la defensa nacional, la seguridad económica, la soberanía alimentaria y la seguridad ambiental, entre otras (p.62)

\section{CONCLUSION}

En la presente revisión del caso práctico, se ha podido determinar la falta de valoración de las pruebas por parte del juez de garantías penales en la audiencia de juzgamiento por contravenciones de tránsito. 
Iustitia Socialis. Revista Arbitrada de Ciencias Jurídicas.

Año V. Vol. V. N³. Edición Especial. 2020-III:

Universidad Regional Autónoma de los Andes

Hecho el depósito de Ley: FA2016000064 ISSN: 2542-3371

FUNDACIÓN KOINONIA (F.K). Santa Ana de Coro, Venezuela

Iyo Alexis Cruz-Piza; Lady Carolina Flores-Córdova

Las sentencias por contravenciones de tránsito no son apelables, salvo las que tienen como sanción penas privativas de libertad, por lo que, el sentenciado no puede ejercer su derecho a recurrir el fallo quedando en estado de indefensión.

\section{FINANCIAMIENTO}

No monetario.

\section{AGRADECIMIENTO}

A la Universidad Regional Autónoma de Los Andes, Babahoyo; por motivar el desarrollo de la Investigación.

\section{REFERENCIAS CONSULTADAS}

Asamblea Nacional del Ecuador. (2010). Código Orgánico de Organización Territorial Autonomía y Descentralización. [Organic Code of Territorial Organization, Autonomy and Decentralization]. Quito - Ecuador: LEXIS FINDER

Asamblea Nacional Constituyente. (20 de octubre de 2008). Constitución de la República del Ecuador. Derechos de protección, 1-80. [Constitution of the Republic of Ecuador. Protection rights, 1-80]. Quito, Pichincha, Ecuador: Registro Oficial.

Bernal-Torres, C. A. (2006). Metodología de la investigación. [Research methodology] Recuperado de https://n9.cl/8txy.

Cevallos Sánchez, G., \& Alvarado Moncada, Z. (2018). Tutela judicial efectiva y la relación con el principio de inmediación. Universidad y Sociedad, 10(1), 168-173. Recuperado de https://n9.cl/1v4v1

Código Orgánico Integral Penal. Decreto Legislativo. [Comprehensive Criminal Organic Code]. Registro Oficial 180 del 10 de febrero de 2014

Constitución de la República del Ecuador. [Constitution of the Republic of Ecuador] Decreto Legislativo. Registro Oficial 449 del 20 de octubre de 2008.

Corte Interamericana de Derechos Humanos. [Inter-American Court of Human Rights](02 de 02 de 2001). Caso Baena y otro. Vs. Panamá. San José, Costa Rica. 


\author{
lustitia Socialis. Revista Arbitrada de Ciencias Jurídicas. \\ Año V. Vol. V. N³. Edición Especial. 2020-III: \\ Universidad Regional Autónoma de los Andes \\ Hecho el depósito de Ley: FA2016000064 \\ ISSN: 2542-3371 \\ FUNDACIÓN KOINONIA (F.K). Santa Ana de Coro, Venezuela \\ Iyo Alexis Cruz-Piza; Lady Carolina Flores-Córdova
}

Corte Constitucional para el periodo de transición. (2012). Genealogía de la justicia constitucional ecuatoriana. [Genealogy of Ecuadorian constitutional justice]. (D.Martínez Molina, Ed.) Quito: Centro de Estudios Constitucional y Difusión del Derecho Constitucional (CEDEC).

Hora, D. L. (12 de Septiembre de 2019). Diario La Hora. Obtenido de https://n9.cl/e70vg

Gómez, M. (2006). Introducción a la metodología de la investigación científica. [Introduction to the methodology of scientific research]. Recuperado de: https://n9.cl/bm0s

Marcheco-Acuña, B. (2017) La tutela cautelar en los procesos contra la administración pública en Ecuador. [The interim protection in processes against the public administration in Ecuador]. Revista Derecho, 3(1),263-285. http://dx.doi.org/10.4067/S0718

Palella Stracuzzi, S. \& Martins Pestana, F. (2012). Metodología de la investigación cuantitativa. [Quantitative research methodology]. Fondo editorial de la Universidad Pedagógica Libertador. Caracas, Venezuela.

Sentencia N.o 090-15-SEP-CC, Caso N. 0 1567-13-EP (Corte Constitucional 25 de Marzo de 2015).

Sentencia No. 045-15-SEP-CC, Caso No. 1055-11-EP (Corte Constitucional 15 de Febrero de 2015).

Zambrano-Noles, S. (2016) El acceso a la justicia y la tutela judicial efectiva en relación con la seguridad ciudadana en Ecuador. [Access to Justice and to Effective Judicial Protection with Respect to Citizen Security in Ecuador]. Revista Tiamelaua.9(39),58-78. Recuperado en https://n9.cl/2cu8

(C2020 por los autores. Este artículo es de acceso abierto y distribuido según los términos y condiciones de la licencia Creative Commons Atribución-NoComercial-Compartirlgual 4.0 Internacional (CC BY-NC-SA 4.0)

(https://creativecommons.org/licenses/by-nc-sa/4.0/). 